\title{
Differential Treatment Response, Neuroinflammation, and Psychosis Associated with Chromosome Deletion
}

\author{
By Eric Hollander, MD
}

There is substantial evidence that obsessivecompulsive disorder (OCD) symptoms can be grouped into a series of discrete dimensions, and some evidence that not all OCD symptom dimensions respond equally well to treatment interventions. The response of OCD symptom dimensions to 12 weeks of treatment with escitalopram/placebo, was investigated by Dan J. Stein, MD, PhD, and colleagues. Factor analysis of individual Yale-Brown Obsessive-Compulsive Scale items yielded 5 factors (contamination/cleaning, harm/ checking, hoarding/symmetry, religious/sexual, and somatic/hypochondriacal). While escitalopram exhibited good efficacy across the range of OCD symptom dimensions, hoarding/symmetry was associated with a poorer treatment response. Hoarding/symmetry may be particularly characteristic of an early-onset group of OCD patients, with the involvement of neurotransmitters other than serotonin. Further work is needed to fully delineate the subtypes of $O C D$, and their correlates with underlying psychobiology and treatment responsivity. A greater understanding of subfactors within various symptom domains may eventually lead to greater understanding of differential treatment outcomes.

Multiple lines of evidence suggest that inflammation and glutamate dysfunction contribute to the pathophysiology of depression. Jonas Hannestad, MD, PhD, and colleagues reviewed how these two systems may interact. Excess levels of inflammatory mediators occur in a subgroup of depressed patients. Acute experimental activation of the immune system with endotoxin and of chronic activation during interferon- $\alpha$ treatment show that inflammation can cause depression. Peripheral inflammation leads to microglial activation, which could interfere with excitatory amino acid metabolism, leading to inappropriate glutamate receptor activation. Loss of astroglia, a feature of depression, upsets the balance of anti- and pro-inflammatory mediators and further impairs the removal of excitatory amino acids. Microglia activated by excess inflammation, astroglial loss, and inappropriate glutamate receptor activation may ultimately disrupt the delicate balance of neuroprotective versus neurotoxic effects in the brain, potentially leading to depression. Thus, psychiatric disorders, such as depression, may benefit from a greater understanding of the role of inflammation in its pathophysiology. Medical specialties, such as rheumatology, have recently benefited from greatly expanded treatment options for inflammation.

Varenicline has demonstrated efficacy in the promotion of smoking cessation. However, from the time of initial trials, it has been associated with significant psychiatric adverse effects. Andres J. Pumariega, MD, and colleagues describe a case where mixed mood and psychotic distur-

Dr. Hollander is the editor of this journal, Esther and Joseph Klingenstein Professor and Chairman of Psychiatry at the Mount Sinai School of Medicine, and director of the Seaver and New York Autism Center of Excellence in New York City. 
bance developed in an individual with a history of depression and family history of bipolar disorder. Based on this case, the authors hypothesize a possible mechanism of action for these adverse effects and preventive measures that could be undertaken in its effective use.

Genetic factors are known to contribute to the development of schizophrenia and related psychoses. Cytogenetic abnormalities have been occasionally found in patients with psychotic disorders and thus helped identify candidategene contributors for these conditions. Milen Velinov, MD, PhD, and colleagues describe a case with mental retardation and anxiety disorder with onset in mid-childhood. In his early 20s, the patient begin exhibiting various psychotic manifestations, including delusions and hallucinations. His psychotic symptoms were difficult to control with psychotropic medications. The family history was negative for psychiatric disorders. This patient was found to have a $6.2 \mathrm{Mb}$ deletion of the terminal portion of the short arm of chromosome 12 that was characterized using fluorescent in situ hybridization and microarray comparative genomic hybridization analysis. The maternal chromosomes were normal, but the paternal chromosomes could not be tested. To date, such a chromosomal abnormality has not been described in association with schizophrenia/psychosis. This case suggests that psychosis-associated gene(s) may be located in the terminal region of the short arm of chromosome 12. These cytogenetic abnormalities seem to be making a greater contribution to the understanding of various neuropsychiatric disorders, ranging from autism to schizophrenia.

Individuals with anxiety disorders often remain symptomatic despite treatment with a first-line pharmacologic agent. More research examining various augmentation strategies to improve outcomes is needed. In an open-label, 8-week augmentation study, Elizabeth A. Hoge, MD, and colleagues examined the efficacy and tolerability of aripiprazole in adult outpatients with generalized anxiety disorder or panic disorder who remained symptomatic despite adequate. Aripiprazole augmentation was associated with a significant reduction in Clinical Global Impressions-Severity scores. Some subjects discontinued due to sedation, chest discomfort, or restlessness. This provides preliminary evidence that aripiprazole may be a useful augmentation strategy for individuals with treatment-resistant generalized anxiety disorder or panic disorder. Treatment resistance clearly is an important factor in academic care settings.

These themes of identifying discrete symptom domains, understanding the role of neuroinflamation in neuropsychiatry, and identifying cytogenetic abnormalities are assuming greater interest and importance in current practice. For example, the Diagnostic and Statistical Manual of Mental Disorders, Fifth Edition process is attempting to incorporate dimensional approaches into our classification system. This is partly based on the notion that symptom dimensions cut across different diagnosis, and often are the focus of targeted treatments by clinicians. With increased focus on symptom dimensions, we will be in a position to understand more about the basic neurocircuitry and differential treatment response that underlies discrete symptom subfactors. Likewise, inflammatory mechanisms play an increasing role across various medical specialties, from cardiology to rheumatology to diabetes. It is not unexpected then that inflammation might become the focus of increased interest in neuropsychiatrtic disorders ranging from Alzheimer's disease and schizophrenia to autism.

Finally, with a rapidly increasing technology for DNA chips, copy number variations have been discovered in an increasing number of individuals who have rare cytogenetic deletions or duplications that directly cause the development of conditions ranging from autism and psychosis to mental retardation. This month's CNS Spectrums addresses some of these emerging themes. CNS 